\title{
O Efeito de Carga Emocional e Intervalo De Retenção na Memória De Testemunhas Oculares
}

\section{[The Role of Arousal and Retention Interval on Eyewitness Memory]}

\author{
Renan Benigo Saraiva ${ }^{1 *}$, Lara Souza ${ }^{2}$, Raiane Nogueira $^{2}$, Letícia Coelho ${ }^{2}$, Luciana Alarcão $^{2}$ \\ ${ }^{1}$ Departamento de Psicologia, University of Portsmouth, Reino Unido. \\ ${ }^{2}$ Instituto de Psicologia, Universidade de Brasilia, Brasil. \\ *Correspondência deve ser endereçada à Renan Benigno Saraiva, Department of Psychology, \\ Faculty of Science, University of Portsmouth, King Henry Building, Portsmouth PO12DY, UK \\ (email: renan.saraiva@ port.ac.uk)
}

Resumo. Presenciar um crime é uma situação de grande estresse e com forte carga emocional, fatores diretamente relacionados à qualidade do relato de testemunhas oculares. Em dois experimentos, participantes assistiram a eventos com diferentes cargas emocionais (neutro, assalto ou assasinato) e forneceram seus relatos após diferentes intervalos de retenção (teste imediato, 7 dias, 14 dias ou 21 dias). Os resultados mostraram que a carga emocional não teve efeito na memória de testemunhas para aspectos do crime e características do criminoso. O evento com maior carga emocional teve um efeito benéfico na memória após um intervalo de 7 dias, mas tal efeito não ocorreu em intervalos mais longos. Implicações teóricas e práticas relacionadas à interpretação do relato de testemunhas são discutidas.

Palavras-chave: Testemunho ocular; Carga emocional; Intervalo de retenção; Memória

Essa é a versão Post-Print do artigo final (após comentário dos revisores) aceito para publicação na revista Teoria e Pesquisa.

This is the final Post-Print version of the article (after peer-review) accepted for publication at Teoria e Pesquisa. 


\begin{abstract}
Witnessing a crime can be a highly stressful situation, eliciting arousal levels that may affect eyewitness memory performance. In two experiments, participants watched a mock crime with varying arousal intensities (neutral, robbery, and murder), and provided reports after varying retention intervals (immediate testing, 7 days, 14 days or 21 days). Results showed that arousal did not have a significant main effect on eyewitness memory recall. Eyewitness memory performance was stronger for the event with higher arousal only after an 7 days retention interval, but performance was comparable across all arousal conditions in longer retention intervals. Theoretical and practical implications related to the evaluation of eyewitness testimony are discussed.
\end{abstract}

Keywords: Eyewitness testimony; Arousal; Retention interval; Memory 
Testemunhas oculares possuem um papel fundamental em investigações criminais e processos judiciais, especialmente em situações que carecem de evidências físicas (e.g., gravações de vídeo ou amostras de DNA). Inúmeras decisões sobre a culpa ou inocência de determinado suspeito são influenciadas pelo relato de testemunhas, seja por meio de descrições do ocorrido seja pela identificação do infrator. Contudo, o estudo sistemático do testemunho ocular demonstra que testemunhas são propensas a erros em seus relatos, devido a fatores sociais e cognitivos (Lindsay, Ross, Read, \& Toglia, 2013; Saraiva \& Castilho, 2018; Saraiva et al., 2015). A falibilidade da memória de testemunhas é considerada a principal causa de prisões injustas de inocentes, desempenhando um papel em mais de $70 \%$ dos casos revistos usando testes de DNA (The Innocence Project, n.d.). Sendo assim, diversas pesquisas dedicam-se à investigação de fatores que influenciam a memória de testemunhas, visando estimar a qualidade de seus relatos em função das circunstâncias do crime. O presente estudo insere-se nesse escopo e tem como objetivo investigar o efeito do grau de violência de um crime no relato de testemunhas oculares.

\section{Testemunhas oculares e carga emocional}

Aspectos relacionados à carga emocional de crimes têm sido extensivamente investigados na literatura do testemunho ocular, incluindo pesquisas que abordam consequências cognitivas ao testemunhar um evento ansiogênico (Christianson, 1992; Deffenbacher, Bornstein, Penrod, \& McGorty, 2004). Apesar da existência de inúmeras pesquisas na área, ainda existe uma considerável controvérsia sobre o efeito de cargas emocionais na memória de testemunhas, e evidências contraditórias dificultam a obtenção de um consenso. Por um lado, pesquisas apontam que eventos ansiogênicos facilitam a codificação do evento, de forma que a carga emocional funciona como uma forte pista mnemónica e como um elemento direcionador da atenção (Heuer 
\& Reisberg, 1992; Woolnough \& MacLeod, 2001). Em outros casos a simulação de eventos com forte carga emocional interfere na recordação de informações relacionadas ao evento, resultando em uma melhor recordação para eventos emocionalmente neutros (Roemer et al., 1998;

Southwick et al., 1997).

Autores sugerem que tais discrepâncias se devem a inconsistências na quantidade e no tipo de carga emocional utilizadas entre diferentes estudos, além de variações nos métodos empregados para eliciar a carga emocional (Brown, 2003; Sauerland et al., 2016). De fato, grande parte dos estudos na área comparam um evento neutro com um único evento com carga emocional, impossibilitando a investigação de efeitos relacionados a eventos com maior ou menor carga emocional. Além disso, a maioria dos estudos sobre testemunhas oculares utilizam testes imediatos, de forma que a testemunha realiza uma identificação ou fornece seu relato em alguns minutos ou horas depois do evento. A presente pesquisa visa explorar essas lacunas, testando o desempenho de testemunhas em eventos com diferentes cargas emocionais, além de investigar possíveis interações decorrentes de diferentes intervalos de retenção.

\section{Informações centrais e informações periféricas}

Algumas contradições relacionadas ao efeito da carga emocional no testemunho ocular podem ser explicadas pelo tipo de informação que está sendo avaliado. Estudos demonstram que eventos com cargas emocionais geralmente beneficiam a memória para aspectos centrais do evento, em detrimento de informações periféricas da cena (Houston, Clifford, Phillips, \& Memon, 2013). Em uma revisão da literatura, Christianson (1992) argumenta que o aspecto emocional restringe o tipo de informação que é melhor percebido, de forma que características centrais da cena são lembradas mais facilmente, enquanto a memória para informações periféricas é prejudicada (Easterbrook, 1959). Apesar de muitos estudos distinguirem 
informações centrais e periféricas do evento, ainda existe uma inconsistência nas definições do que é considerado central ou periférico (Houston et al., 2013). Algumas definições se baseiam principalmente na posição espacial da informação, classificando como centrais aquelas que se encontram em um plano mais central no campo de visão, enquanto informações no fundo são consideradas periféricas (Christianson \& Loftus, 1991). Uma definição mais comum na área leva em consideração a relação entre a informação e o enredo do evento, classificando como informações centrais aquelas que estão diretamente relacionadas ao estímulo estressor, e classificando como periféricas aquelas informações que não são relevantes para o evento principal (Heuer \& Reisberg, 1992).

Diversas evidências apoiam a existência de efeitos diferenciados para informações centrais e periféricas, apontando que informações centrais são lembradas com maior facilidade em eventos com carga emocional. Burke, Heuer, e Reisberg (1992), por exemplo, encontraram que a memória de testemunhas foi mais acurada para aspectos centrais (tanto conceitual quanto espaço-temporalmente) de um evento emocional. O mesmo padrão se repete em estudos de laboratório (Ibabe \& Sporer, 2004), estudos de identificação de fotos (Safer, Christianson, Autry, \& Österlund, 1998), experiências autobiográficas traumáticas (Wessel \& Merckelbach, 1994), e em estudos de casos reais (Christianson \& Hübinette, 1993).

Apesar de a maioria dos estudos demonstrarem que informações centrais são mais facilmente lembradas em eventos com carga emocional, ainda existem resultados que não corroboram esse efeito. Em um estudo conduzido por Morgan et al. (2004), por exemplo, soldados foram submetidos a um interrogatório estressante como parte de um treinamento de resistência a interrogatórios. Neste estudo os participantes foram divididos em condições de maior ou menor estresse. Participantes que foram submetidos ao interrogatório de maior estresse falharam com maior frequência em uma tarefa de identificação do interrogador, quando 
comparado com participantes na condição de menor estresse. Outros estudos também encontraram que indivíduos em situações estressoras são menos aptos a reconhecer o alvo em tarefas de identificação (Houston et al., 2013). Esses resultados sugerem que a simples distinção entre informações centrais e periféricas não é suficiente para explicar como a carga emocional afeta a memória de testemunhas oculares.

\section{Modelos teóricos de carga emocional e testemunho ocular}

Muitas teorias postulam que o efeito da carga emocional na memória depende da intensidade do estímulo emocional. Duas principais correntes teóricas são as mais mencionadas e utilizadas para explicar o desempenho de testemunhas para eventos com diferentes níveis de carga emocional: a lei de Yerkes-Dodson (1908) e a hipótese de utilização de pistas de Easterbrook (1959). A lei de Yerkes-Dodson postula que o desempenho de testemunhas respeita uma função de U invertido, em que níveis muito baixos e altos de carga emocional geram pior acurácia, enquanto que níveis intermediários de carga emocional geram melhor acurácia (Deffenbacher, 1983, Deffenbacher et al., 2004). A hipótese de utilização de pistas de Easterbrook prediz que a performance aumenta conjuntamente com a carga emocional, mas apenas para os aspectos mais centrais do evento testemunhado, enquanto a memória para elementos periféricos diminui com o aumento da carga emocional (Christianson, 1992). A literatura atual sobre a acurácia de testemunhas não é suficiente para determinar qual teoria é mais robusta, dado que apenas poucos estudos investigaram mais de um evento com carga emocional em seus procedimentos.

O objetivo do primeiro estudo foi investigar a memória de testemunhas oculares para eventos com três níveis crescentes de carga emocional, avaliando tanto a recordação de informações gerais do evento quanto a identificação por conjuntos de fotos. Tal como revisado 
anteriormente, estabelecer predições sobre esse efeito de forma direta não é elementar, tendo em vista contradições na literatura. Entretanto, não foi esperado que o evento com maior carga emocional deste estudo fosse comparável ao nível de estresse obtido em outros estudos (Morgan et al., 2004), de forma que não foi esperado que a carga emocional no presente estudo alcançasse efeitos prejudiciais à memória. Sendo assim, foi esperado que a memória de testemunhas para informações centrais seria mais acurada conforme o aumento da carga emocional do evento, enquanto a memória para informações periféricas seria prejudicada.

\section{Experimento 1}

\section{Método}

\section{Delineamento}

Foi utilizado um delineamento entre sujeitos para verificar possíveis diferenças no desempenho de testemunhas para eventos com três cargas emocionais distintas: Controle, Menor Carga Emocional e Maior Carga Emocional. A acurácia de testemunhas oculares foi mensurada por meio de questões sobre características do evento (gerais, centrais e periféricas) e por meio de uma tarefa de identificação por fotos.

\section{Participantes}

Noventa estudantes universitários (60\% mulheres) participaram deste estudo, com idade média de 21 anos $(D P=4,50)$. Os participantes foram convidados diretamente, por meio de ligações ou listas de e-mails de interessados em participar de pesquisas em psicologia.

\section{Materiais}

\section{Inventário de Ansiedade Traço-Estado (IDATE; State-Trait Anxiety Inventory,} Spielberger, 1983). Foi utilizada uma versão adaptada para o Brasil do Inventário de Ansiedade Traço-Estado (IDATE; Biaggio \& Spielberger, 1983). O instrumento foi empregado para avaliar as cargas emocionais dos diferentes eventos apresentados aos participantes. O IDATE é um 
instrumento de auto-avaliação com 20 items (e.g., "Sinto-me calmo"), respondidos em uma escala de quatro pontos: 1 - absolutamente não, 2 - um pouco, 3 - bastante, e 4 - muitíssimo. Para os propósitos deste estudo apenas a versão "estado" foi utilizada, em que os itens são respondidos de acordo com a forma como o participante se sente em um dado momento. Um único fator relacionado a sentimentos negativos foi utilizado $(\alpha=0.92)$.

Eventos testemunhados. Três vídeos de aproximadamente 1:30min foram elaborados, um para cada condição de carga emocional. A narrativa dos três vídeos consiste em um homem caminhando com uma mulher em um parque, enquanto outras pessoas caminham no fundo. Somente uma cena crítica diferiu entre as três condições, relacionado a um terceiro ator que interage brevemente com a mulher que estava andando no parque, envolvendo uma conversa (condição Controle), um assalto (Menor Carga Emocional) ou um latrocínio (Maior Carga Emocional). Nas cenas com carga emocional o terceiro ator foi o culpado dos crimes e a atriz foi a vítima. Todas as cenas críticas ocorreram nos últimos 30 segundos do vídeo.

Teste de informações do crime. Foi elaborado um questionário relacionado aos detalhes do vídeo, com 16 afirmações relacionadas a informações centrais da cena e 15 relacionadas a informações periféricas. As afirmações incluíram detalhes sobre os três personagens principais do vídeo e ações que ocorreram no fundo. Nomes fictícios foram atribuídos aos atores principais para facilitar a identificação destes no questionário. As questões centrais são aquelas associadas ao evento crítico e os atores envolvidos (e.g., "Paulo tinha cabelo longo"). As questões periféricas consistem em elementos não associados ao evento estressor (e.g., "ciclistas apareceram no vídeo”). Cada afirmação precisava ser julgada como verdadeira ou falsa, acompanhada de uma escala de confiança de $10 \%$ confiante à $100 \%$ confiante.

Tarefa de identificação por fotos. Conjuntos de fotos foram elaborados para serem utilizados em tarefas de identificação de suspeito. Inicialmente todos os participantes receberam 
um conjunto de fotos com oito rostos de homens, apresentados simultaneamente, e sua tarefa era tentar identificar o terceiro ator do vídeo. Os participantes foram instruídos a julgar suas respostas em uma escala de $10 \%$ confiante à $100 \%$ confiante. Após sua primeira resposta, um segundo conjunto de fotos era apresentado com oito fotos novas, e os participantes eram requisitados a repetir a tarefa, mesmo que tenham identificado alguém no conjunto anterior. Nesse momento metade dos participantes receberam um conjunto com o culpado presente e a outra metade recebeu um conjunto com o culpado ausente. Os participantes foram informados em ambos os conjuntos de fotos que o alvo poderia ou não estar entre as fotos.

\section{Procedimento}

Os participantes foram designados a uma de três condições: Controle, Menor Carga Emocional ou Maior Carga Emocional. Inicialmente eles assistiram ao vídeo de sua respectiva condição, projetado em uma tela. Após assistirem ao vídeo os participantes completaram o Inventário de Ansiedade Estado e foram submetidos a uma tarefa distratora logo em seguida, que consistia em encontrar letras "L" misturadas entre letras “T”. Essa tarefa era encerrada após 5 minutos mesmo que o participante não encontrasse todos as letras "L". Após a tarefa distratora os participantes receberam um questionário sobre o vídeo e responderam à tarefa de identificação do suspeito.

\section{Resultados}

As principais análises consistiram majoritariamente em análises de variância (ANOVA) e correlações. As análises inferenciais não apresentaram problemas em relação à normalidade da distribuição, linearidade, homocedasticidade, equivalência de variâncias entre grupos ou outros pressupostos. O teste de Tukey HSD foi utilizado como teste post-hoc nas análises de variância.

\section{Checagem de manipulação de carga emocional}

As análises iniciais visaram comparar os escores do IDATE entre as diferentes condições 
experimentais. Uma ANOVA entre sujeitos revelou uma diferença significativa no nível de sentimentos negativos entre as três condições experimentais, $F(1,85)=19,07, p<0,001, \eta^{2} \mathrm{p}=$ 0,18. Como esperado, participantes na condição de Maior Carga Emocional reportaram mais sentimentos negativos $(M=2.66, D P=0.43)$, seguido dos participantes da condição de Menor Carga Emocional $(M=2.35, D P=0.54)$, e da condição Controle $(M=2.10, D P=0.44)$.

\section{Acurácia para informações do crime e carga emocional}

Foram analisados os efeitos da carga emocional na acurácia e confiança apresentados no teste de informações sobre o crime. Análises descritivas das variáveis de acurácia e confiança por condição e análises de variância comparando desempenho e carga emocional são apresentadas na Tabela 1. A quantidade de acertos para informações gerais foi semelhante entre as três condições de carga emocional, e o mesmo resultado foi encontrado ao avaliar o desempenho específico para informações centrais e informações periféricas. Também não foi encontrada correlação entre acurácia e o fator de sentimentos negativos do IDATE $(r=-0,07, p=0,50)$. Foi encontrada uma correlação moderada entre confiança e acurácia geral $[r(87)=0,36, p<0,001]$, e essa relação foi maior para informações centrais $[r(87)=0,39, p<0,001]$, quando comparado com informações

periféricas $[r(87)=0,27, p<0,001]$. Em conjunto, tais resultados sugerem que o desempenho e a confiança de testemunhas ao relatar informações específicas de um crime não é influenciado pela carga emocional do evento. Adicionalmente, os resultados indicam que a confiança que testemunhas exibem em seu relato possui uma relação moderada com acurácia.

[Inserir Tabela 1]

\section{Acurácia na identificação por fotos e carga emocional}

A proporção de acertos na identificação de fotos com o alvo presente foi de $14 \%$, enquanto a proporção de acertos com o alvo ausente foi de $36 \%$. Não foram encontradas diferenças significativas entre as diferentes condições de carga emocional para acurácia na 
identificação por fotos com alvo presente ou ausente (ver Tabela 2). Não foi encontrada correlação entre confiança e acurácia na tarefa de identificação por fotos com o alvo presente $(r=$ $-0,15, p=0,30)$, mas tal relação foi significativa na identificação com o alvo ausente $(r=0,34, p$ $=0,01)$. De forma semelhante ao observado com informações gerais sobre o crime, tais resultados sugerem que a carga emocional não influencia a capacidade de testemunhas de identificarem um suspeito em um conjunto de fotos. Ademais, a confiança que testemunhas exibem durante uma identificação com o alvo presente não se relaciona com a acurácia geral para esta tarefa, mas tal relação é moderada quando o alvo não está presente no conjunto de fotos.

\section{[Inserir Tabela 2]}

\section{Experimento 2}

O objetivo do Experimento 1 foi testar o efeito de três níveis distintos de carga emocional na memória de testemunhas oculares, para detalhes do crime e para índices de identificação do criminoso. Foi encontrado que os vídeos elaborados com maior carga emocional geraram mais sentimentos de ansiedade e estresse medidos pelo IDATE-Estado. Entretanto, não foram encontradas diferenças significativas para memória sobre o crime entre as diferentes condições de carga emocional, de forma que os participantes apresentaram um desempenho semelhante para informações centrais e periféricas entre as diferentes condições experimentais. Também não foram encontradas diferenças de performance na tarefa de identificação de suspeitos entre os grupos de carga emocional, tanto para tarefas com o alvo presente quanto com o alvo ausente. Estes resultados sugerem que testemunhas oculares possuem um desempenho de memória semelhante para eventos com diferentes cargas emocionais. Por outro lado, no Experimento 1 todas as testemunhas foram testadas de forma imediata, de forma que o teste de reconhecimento ocorreu apenas cinco minutos depois da codificação dos estímulos emocionais. Além de apresentar uma situação que não é comum em casos criminais reais, é possível que tal 
recuperação imediata de um estímulo recém-codificado atenue o efeito da carga emocional na memória.

O intervalo de retenção é uma variável de interesse em estudos sobre testemunhas oculares por diversos motivos. Após presenciarem um crime, testemunhas de crimes reais podem levar de horas a meses para fornecerem seus relatos ou identificarem suspeitos (Behrman \& Davey, 2001; Saraiva et al., 2017). Inúmeras teorias sobre reconhecimento e evocação de memórias sugerem que, em geral, a quantidade e qualidade das informações armazenadas na memória decai com o passar do tempo (Baddeley, 1997; Reyna \& Brainerd, 1995). Esta argumentação é comumente apoiada em estudos sobre o testemunho ocular, e diversos estudos apontam para uma relação negativa entre o desempenho de testemunhas oculares e o intervalo de retenção entre o crime e o relato sobre o ocorrido (Sauer, Brewer, Zweck, \& Weber, 2010; Wetmore et al., 2015; Wixted, Don Read, \& Stephen Lindsay, 2016). Sauer et al. (2010), por exemplo, encontraram que identificações realizadas por testemunhas oculares após três a sete semanas do ocorrido foram muito menos acuradas do que identificações realizadas imediatamente após o crime. Em uma metanálise realizada por Park (2005), foram encontradas interações entre carga emocional e intervalo de retenção para vários tipos de memória. Especificamente, foi encontrado que em intervalos curtos o desempenho de memória é melhor para estímulos com pouca carga emocional, enquanto em intervalos longos a memória é mais acurada para estímulos com muita carga emocional.

O objetivo do Experimento 2 foi testar se o efeito da carga emocional na memória de testemunhas oculares é moderado pelo intervalo de retenção entre o crime e a recordação do crime. Foram utilizados os mesmos materiais elaborados no Experimento 1, porém os testes sobre informações do crime foram realizados 7 dias, 14 dias, ou 21 dias após a exposição ao crime. Foi hipotetizada uma relação negativa entre o intervalo de retenção e memória para informações 
sobre o crime e índices de identificação do suspeito. Adicionalmente, foi esperado que a memória para eventos com maior carga emocional seria mais duradoura após longos intervalos de retenção, enquanto a memória para eventos com menor carga emocional desaparece mais rapidamente após longos intervalos de retenção.

\section{Método}

\section{Delineamento}

Um delineamento fatorial $3 \times 3$ entre sujeitos foi utilizado para testar o desempenho de testemunhas para eventos com três cargas emocionais (Controle, Menor Carga Emocional e Maior Carga Emocional) e três diferentes intervalos de retenção (7 dias, 14 dias ou 21 dias). A acurácia das testemunhas foi mensurada utilizando os mesmos materiais e tarefas do Estudo 1.

\section{Participantes}

Cinquenta e sete estudantes universitários (74\% mulheres) participaram deste estudo, com idade média de 21 anos $(D P=6,30)$, convidados pessoalmente, por telefone ou por listas de emails.

\section{Materiais}

Os vídeos e tarefas de reconhecimento (questionário sobre o crime e conjuntos de fotos) foram os mesmos utilizados no Estudo 1. Não foi necessário incluir tarefas de distração neste estudo tendo em vista os intervalos de retenção utilizados. Também foi decidido pela supressão do Inventário de Ansiedade Traço-Estado, uma vez que o Estudo 1 apresentou evidências para diferentes cargas emocionais dos vídeos utilizados.

\section{Procedimento}

Os participantes foram designados a uma de três condições de carga emocional: (1) Controle; (2) Menor Carga Emocional; (3) Maior Carga Emocional. Após serem alocados em uma condição de carga emocional, os participantes foram designados para uma de três condições 
de intervalo de retenção: 7, 14, ou 21 dias. Dessa forma, cada condição de carga emocional possui participantes de cada um dos três grupos de intervalos de retenção descritos. Inicialmente, os participantes assistiram ao vídeo respectivo de suas condições de carga emocional no laboratório, sendo então informados de que receberiam um link para um survey online em alguns dias. Participantes receberam o e-mail de acordo com sua respectiva condição de intervalo de retenção. O survey online continha o questionário sobre informações do crime e as tarefas de identificação por fotos utilizado no Experimento 1. Também foi requisitada uma breve descrição do vídeo que assistiram, para confirmar que o vídeo correto estava sendo considerado em suas respostas.

\section{Resultados}

\section{Acurácia para informações do crime}

De forma semelhante ao Experimento 1, a acurácia dos participantes nas informações sobre o crime foi equiparável entre as três condições de carga emocional, $F(1,54)=0.55, p=.58$, $\eta_{p}^{2}=0.019$. O mesmo padrão foi encontrado para informações centrais $[F(1,54)=1.63, p=.20$, $\left.\eta_{p}^{2}=0.056\right]$ e informações periféricas $\left[F(1,54)=0.21, p=.81, \eta_{p}^{2}=0.007\right]$. A confiança nas respostas sobre informações do crime também não diferiu significativamente entre as diferentes condições de carga emocional, $F(1,54)=0.26, p=.76, \eta_{\mathrm{p}}^{2}=0.009$. A Tabela 3 apresenta as estatísticas descritivas e ANOVAs comparando a acurácia na tarefa de informações sobre o crime entre as condições de intervalo de retenção. Não foram encontradas diferenças significativas na acurácia sobre informações do crime entre as diferentes condições de intervalo de retenção. Foi encontrado um efeito significativo do intervalo de retenção na confiança de testemunhas oculares para todos os tipos de informações, com maior efeito para informações centrais. Análises de comparação post-hoc usando o teste de Tukey HSD indicaram que a média de confiança da condição de 21 dias $(M=61 \%, D P=17)$ diferiu significativamente da média da condição de 7 
dias $(M=76 \%, D P=12)$, mas nenhuma média diferiu significativamente da condição de 14 dias $(M=65 \%, D P=16)$. Uma ANOVA de duas vias revelou que não houve uma interação significativa entre carga emocional e intervalo de retenção na acurácia para informações sobre o crime $F(2,48)=2.33, p=.06$. Porém, uma análise de contrastes revelou que participantes na condição de muita carga emocional tiveram mais acertos gerais quando comparados com participantes na condição neutra e de pouca carga emocional, tal efeito só ocorreu para intervalo de retenção de 7 dias $F(1,48)=4.24, p=.04$ (ver Figura 1). Não foi encontrada uma interação significativa entre carga emocional e intervalo de retenção para confiança sobre informações do crime $F(2,48)=1.21, p=.32($ ver Figura 2$)$.

[Inserir Tabela 3]

[Inserir Figura 1]

[Inserir Figura 2]

\section{Acurácia para identificação por fotos}

A proporção de acertos geral na identificação de fotos com o alvo presente foi de $3 \%$, já proporção de acertos com o alvo ausente foi de $37 \%$. Não foram encontradas diferenças significativas entre as diferentes condições de carga emocional para acurácia na identificação por fotos com alvo presente $\left[F(1,28)=1.00, p=.38, \eta_{p}^{2}<0.06\right]$ ou ausente $[F(1,25)=0.73, p=.48$ $\left.\eta_{\mathrm{p}}^{2}=0.06\right]$. A Tabela 4 apresenta que também não foram encontradas diferenças significativas entre as condições de intervalo de retenção e acurácia na tarefa de identificação por fotos. De forma semelhante ao Experimento 1, não foi encontrada correlação entre confiança e acurácia na tarefa de identificação por fotos com o alvo presente $(r=-.13, p=.49)$ ou com o alvo ausente $(r$ $=-.20, p=.31)$

[Inserir Tabela 4]

\section{Discussão}


O objetivo deste estudo foi investigar o efeito de diferentes níveis de carga emocional na memória de testemunhas oculares, assim como testar possíveis interações entre carga emocional e intervalo de retenção. O desempenho das testemunhas foi analisado por meio de questões sobre informações gerais sobre o crime e uma tarefa de identificação por fotos. Os resultados revelaram que o desempenho para informações gerais sobre o crime não foi influenciado pelos diferentes níveis de carga emocional, de forma que a memória das testemunhas sobre o crime foi semelhante para eventos neutros, com pouca carga emocional ou muita carga emocional. Também foi encontrado o mesmo efeito ao avaliar separadamente o desempenho para informações centrais ou periféricas do crime. Tais evidências sugerem que o nível de carga emocional não afeta substancialmente o relato de testemunhas oculares em situações em que o relato é obtido de forma imediata. Apesar de contradizer nossa hipótese inicial, diversos estudos apontam para uma complexa relação entre a carga emocional e a memória de testemunhas oculares. Em uma primeira metanálise conduzida por Deffenbacher (1983), foi encontrado que 10 estudos indicaram que a carga emocional aumenta ou não afeta a memória de testemunhas, enquanto 11 outros estudos sugeriram que a memória é prejudicada pela carga emocional. Em contraposição, outra revisão realizada por Christianson (1992) argumenta que a carga emocional facilita a memória de testemunhas oculares, mas somente para aspectos centrais do crime. Uma metanálise mais recente apresenta mais evidências de que a carga emocional prejudica a memória de testemunhas, tanto para relatos do crime quanto para identificação de suspeitos (Deffenbacher et al., 2004)

A complexa relação entre carga emocional e memória no testemunho ocular pode ser explicada, em parte, pelo papel de diversas variáveis moderadoras. Deffenbacher et al. (2004), por exemplo, encontrou que o efeito negativo da carga emocional na memória é maior para tarefas de identificação em que o suspeito está presente, comparado com autos de identificação 
em que o suspeito não está presente. O efeito da carga emocional na memória também foi maior em estudos que utilizaram uma simulação real de testemunho ocular, comparado com estudos que utilizaram outros métodos. De fato, estudos que eliciam cargas emocionais mais fortes tendem a gerar uma resposta defensiva (i.e., aversão ao estímulo; Deffenbacher, 1994), enquanto manipulações que geram menor carga emocional tendem a eliciar uma resposta de orientação ao estímulo aversivo (Burke et al., 1992; Christianson, 1992; Levine \& Edelstein, 2009).

Os resultados obtidos no presente estudo não corroboram tal predição, uma vez que a memória das testemunhas não diferiu entre condições de maior ou menor nível de carga emocional. É possível que tal efeito tenha ocorrido devido a outros moderadores que não foram manipulados nos experimentos que conduzimos. Um destes possíveis moderadores é o tempo de exposição que as testemunhas tiveram aos estímulos apresentados. Em nossa manipulação, todas as testemunhas observaram as características dos alvos principais nos vídeos por um minuto, antes de serem expostos aos 30 segundos finais em que a manipulação de carga emocional ocorreu. Tal situação reflete casos que ocorrem no cotidiano, em que vítimas ou testemunhas possuem uma chance de observar características de um criminoso antes da realização do crime. Não obstante, é possível que a codificação inicial diminua a relevância da carga emocional na memória do crime, uma vez que parte das informações sobre as características do criminoso já foram codificadas (Bornstein, Deffenbacher, Penrod, \& McGorty, 2012; Memon, Hope, \& Bull, 2003). No estudo realizado por Dutton e Carroll (2001), foi encontrado que eventos com menor carga emocional foram melhor recordados comparado com eventos neutros e com maior carga emocional, e os estímulos utilizados não apresentaram informações que eram comuns a todas as condições.

No Experimento 1, pode ser argumentado que parte da ausência de efeito da carga emocional na memória de testemunhas deve-se ao fato de o teste ter ocorrido de forma imediata, 
tornando a memória das testemunhas forte o suficiente para anular o efeito de diferentes cargas emocionais (Park, 2005). No Experimento 2 a memória de testemunhas foi testada para três intervalos de retenção distintos (7 dias vs 14 dias vs 21 dias), e não foram encontradas fortes evidências de uma interação entre intervalo de retenção e carga emocional. Entretanto, foi encontrado que em intervalos de retenção curtos (7 dias) a memória para eventos com maior carga emocional foi mais forte, e este efeito não se manteve em intervalos de retenção mais longos. Diversos estudos apontam para uma interação entre intervalo de retenção e carga emocional e indicam que pouca carga emocional gera melhor memória de curto prazo, enquanto muita carga emocional gera melhor memória de longo prazo (Kleinsmith \& Kaplan, 1963, 1964). Uma metanálise identificou um tamanho de efeito moderado para tal interação $(d=0,77$; Park, 2005). Porém, nesta metanálise os resultados avaliados foram restritos a intervalos de retenção entre dois minutos a uma semana, impossibilitando a análise da interação entre carga emocional e intervalos de retenção mais longos. Os resultados obtidos no presente estudo sugerem que estímulos com maior carga emocional facilitam a recordação em intervalos curtos, mas tal efeito desaparece após longos intervalos. É possível que tal efeito ocorra devido ao decaimento natural da memória, de forma que o desempenho de testemunhas oculares após longos intervalos independe da carga emocional do ocorrido (Ebbesen \& Rienick, 1998; Gold, Murray, Sekuler, Bennett, \& Sekuler, 2005).

O presente estudo possui algumas limitações. Conforme o esperado, o Experimento 1 revelou que os participantes experienciaram mais sentimentos negativos ao assistirem o vídeo de maior carga emocional, seguido pelo vídeo de menor carga emocional e do vídeo neutro. Entretanto, este resultado foi obtido por meio de um instrumento de auto-relato (Inventário de Ansiedade Traço-Estado, IDATE; Spielberger, 1983), e o uso exclusivo de medidas subjetivas de carga emocional não permite uma inspeção objetiva de respostas fisiológicas que possam 
modular desempenhos de memória (Hellhammer \& Schubert, 2012). Adicionalmente, é importante ressaltar que o paradigma utilizado neste estudo não corresponde a uma situação estritamente naturalística (e.g., assistir vídeos vs. presenciar um crime), apesar de a utilização de métodos naturalísticos apresentar outras dificuldades metodológicas (Yuille, 2013). Também existem limitações relacionadas às medidas utilizadas para testar a memória das testemunhas oculares. Por exemplo, neste estudo não foram utilizadas tarefas de recordação livre (Hope, Gabbert, Fisher, \& Jamieson, 2014), a tarefa de identificação por fotos foi realizada apenas de forma simultânea, e métodos de apresentação sequencial não foram investigados (Kneller, Memon, \& Stevenage, 2001; Sporer, 1993).

\section{Conclusão}

Não foram encontradas evidências de que a carga emocional de diferentes crimes afeta substancialmente a memória de testemunhas oculares. Os participantes deste estudo tiveram um desempenho equivalente de memória ao assistirem a vídeos neutros (diálogo entre dois atores), pouca carga emocional (assalto) ou muita carga emocional (assassinato). O desempenho em uma tarefa de identificação do suspeito também não foi afetado pela carga emocional. De forma geral, tais resultados sugerem que a memória de testemunhas oculares não é prejudicada nem facilitada pela carga emocional em situações de curto intervalo de retenção. Entretanto, testemunhas do crime com maior carga emocional tiveram uma memória mais acurada do crime na condição com intervalo de retenção de 7 dias. Tal efeito não ocorre com intervalos de retenção maiores que 14 dias. Assim, a memória para eventos com forte carga emocional parece facilitar a memória após um intervalo curto de retenção, mas tal efeito desaparece conforme este intervalo aumenta. Importantes implicações teóricas podem ser obtidas ao avaliar a interação entre carga emocional e intervalo de retenção, revelando possíveis associações entre o decaimento de memória e a valência de estímulos codificados. Além disso, uma melhor compreensão destes fatores pode 
auxiliar profissionais do sistema de justiça criminal que buscam estimar a acurácia de testemunhas oculares.

\section{Referências}

Baddeley, A. D. (1997). Human memory: Theory and practice. East Sussex: Psychology Press.

Behrman, B. W., \& Davey, S. L. (2001). Eyewitness identification in actual criminal cases: An archival analysis. Law and Human Behavior, 25(5), 475-491. doi:

10.1023/A:1012840831846

Biaggio, A., \& Spielberger, C. D. (1983). Inventário de ansiedade traço-estado, Idate-C Manual. Rio de Janeiro, RJ: Centro de Psicologia Aplicada.

Bornstein, B. H., Deffenbacher, K. A., Penrod, S. D., \& McGorty, E. K. (2012). Effects of exposure time and cognitive operations on facial identification accuracy: a meta-analysis of two variables associated with initial memory strength. Psychology, Crime \& Law, 18(5), 473-490. doi: 10.1080/1068316X.2010.508458

Brown, J. M. (2003). Eyewitness memory for arousing events: putting things into context. Applied Cognitive Psychology, 17(1), 93-106. doi: 10.1002/acp.848

Burke, A., Heuer, F., \& Reisberg, D. (1992). Remembering emotional events. Memory \& Cognition, 20(3), 277-290. doi: 10.3758/BF03199665

Christianson, S. A. (1992). Emotional stress and eyewitness memory: A critical review. Psychological Bulletin, 112(2), 284-309. doi: 10.1037/0033-2909.112.2.284

Christianson, S. A., \& Hubinette, B. (1993). Hands up! A study of witnesses and memories associated with bank robberies. Applied Cognitive Psychology, 7, 365-379. doi: 10.1002/acp.2350070502

Deffenbacher, K. A. (1983). The influence of arousal on reliability of testimony. Em S. M. A. Lloyd-Boystock, \& B. R. Clifford (Eds.), Evaluating witness evidence: Recent psychological 
research and new perspectives (pp. 235-251). John Wiley \& Sons.

Deffenbacher, K. A. (1994). Effects of arousal on everyday memory. Human Performance, 7(2), 141-161. doi: 10.1207/s15327043hup0702_3

Deffenbacher, K. A., Bornstein, B. H., Penrod, S. D., \& McGorty, E. K. (2004). A meta-analytic review of the effects of high stress on eyewitness memory. Law and Human Behavior, 28(6), 687-706. doi: 10.1007/s10979-004-0565-x

Dutton, A., \& Carroll, M. (2001). Eyewitness testimony: Effects of source of arousal on memory, source-monitoring, and metamemory judgments. Australian Journal of Psychology, 53(2), 83-91. doi: 10.1080/00049530108255128

Easterbrook, J. A. (1959). The effect of emotion on cue utilization and the organization of behavior. Psychological Review, 66, 183-201. doi: 10.1037/h0047707

Ebbesen, E. B., \& Rienick, C. B. (1998). Retention interval and eyewitness memory for events and personal identifying attributes. The Journal of Applied Psychology, 83(5), 745-762. doi: 10.1037/0021-9010.83.5.745

Levine, L. J., \& Edelstein, R. S. (2009). Emotion and memory narrowing: A review and goalrelevance approach. Cognition and Emotion, 23, 178-220. doi:

$10.1080 / 02699930902738863$

Gold, J. M., Murray, R. F., Sekuler, A. B., Bennett, P. J., \& Sekuler, R. (2005). Visual memory decay is deterministic. Psychological Science, 16(10), 769-774. doi: 10.1111/j.14679280.2005.01612.x

Heuer, F., \& Reisberg, D. (1992). Emotion, arousal and memory for detail. In S. A. Christianson (Ed.). The handbook of emotion and memory: Research and theory (pp. 151-180). Hillsdale, NJ: Erlbaum.

Hope, L., Gabbert, F., Fisher, R. P., \& Jamieson, K. (2014). Protecting and enhancing eyewitness 
memory: The impact of an initial recall attempt on performance in an investigative interview. Applied Cognitive Psychology, 28(3), 304-313. doi:10.1002/acp.2984

Houston, K. A., Clifford, B. R., Phillips, L. H., \& Memon. A. (2013). The emotional eyewitness: The effects of emotion on specific aspects of eyewitness recall and recognition performance. Emotion, 13(1), 118-128. doi: 10.1037/a0029220

Ibabe, I., \& Sporer, S. L. (2004). How you ask is what you get: On the influence of question form on accuracy and confidence. Applied Cognitive Psychology, 18, 711-726. doi: 10.1002/acp. 1025

Innocence Project (n.d.). Recuperado de http://www.innocenceproject.org/

Kneller, W., Memon, A., \& Stevenage, S. (2001). Simultaneous and sequential lineups: Decision processes of accurate and inaccurate eyewitnesses. Applied Cognitive Psychology, 15(6), 659-671. doi:10.1002/acp.739

Lindsay, R. C. L., Ross, D. F., Read, J. D., \& Toglia, M. P. (2013). The handbook of eyewitness psychology: Volume II: Memory for people. Taylor \& Francis.

Memon, A., Hope, L., \& Bull, R. (2003). Exposure duration: Effects on eyewitness accuracy and confidence. British Journal of Psychology, 94(3), 339-354. doi:

$10.1348 / 000712603767876262$

Morgan, C. A., Hazlett, G., Doran, A., Garrett, S., Hoyt, G., Thomas, P., ... \& Southwick, S. M. (2004). Accuracy of eyewitness memory for persons encountered during exposure to highly intense stress. International Journal of Law and Psychiatry, 27(3), 265-279.

Park, J. (2005). Effect of arousal and retention delay on memory: a meta-analysis. Psychological Reports, 97(2), 339-355. doi: 10.2466/pr0.97.2.339-355

Reyna, V. F., \& Brainerd, C. J. (1995). Fuzzy-trace theory: An interim synthesis. Learning and Individual Differences, 7(1), 1-75. doi:10.1016/1041-6080(95)90031-4 
Roemer, L., Litz, B., Orsillo, S. M., Ehlich, P. J., \& Friedman, M. J. (1998). Increases in retrospective accounts of war-zone exposure over time: The role of PTSD symptom severity. Journal of Traumatic Stress, 11(3), 597-605. doi: 10.1023/A:1024469116047

Safer, M. A., Christianson, S. Å., Autry, M. W., \& Österlund, K. (1998). Tunnel memory for traumatic events. Applied Cognitive Psychology, 12(2), 99-117. doi: 10.1023/A:1024469116047

Saraiva, R., \& Castilho, G. M. (2018). Psicologia do testemunho ocular: Aplicações no contexto forense e criminal. São Paulo: Juruá.

Saraiva, R. B., Castilho, G. M. de, Nogueira, R. N., Coelho, L. de A. M., Alarcão, L. C. P., \& Lage, J. (2017). Memória de testemunhas oculares para faces em casos criminais: Uma análise de arquivo de retratos falados positivos. Estudos de Psicologia (Natal), 22(3), 247256. doi: $10.5935 / 1678-4669.20170025$

Sauer, J., Brewer, N., Zweck, T., \& Weber, N. (2010). The effect of retention interval on the confidence-accuracy relationship for eyewitness identification. Law and Human Behavior, 34(4), 337-347. doi:10.1007/s10979-009-9192-x

Sauerland, M., Raymaekers, L. H. C., Otgaar, H., Memon, A., Waltjen, T. T., Nivo, M., ... \& Smeets, T. (2016). Stress, stress-induced cortisol responses, and eyewitness identification performance. Behavioral Sciences \& the Law, 34(4), 580-594. doi: 10.1002/bs1.2249

Southwick, S. M., Morgan III, C. A., Nicolaou, A. L., \& Charney, D. S. (1997). Consistency of memory for combat-related traumatic events in veterans of operation desert storm. American Journal of Psychiatry, 154, 173-177. doi: 10.1176/ajp.154.2.173

Sporer, S. L. (1993). Eyewitness identification accuracy, confidence, and decision times in simultaneous and sequential lineups. The Journal of Applied Psychology, 78(1), 22. doi: 10.1037/0021-9010.78.1.22 
Wetmore, S. A., Neuschatz, J. S., Gronlund, S. D., Wooten, A., Goodsell, C. A., \& Carlson, C. A. (2015). Effect of retention interval on showup and lineup performance. Journal of Applied Research in Memory and Cognition, 4(1), 8-14. doi: 10.1016/j.jarmac.2014.07.003

Wessel, I., \& Merckelbach, H. (1994). Characteristics of traumatic memories in normal subjects. Behavioural and Cognitive Psychotherapy, 22(04), 315-324. doi:

$10.1017 / \mathrm{S} 1352465800013199$

Wixted, J. T., Don Read, J., \& Stephen Lindsay, D. (2016/6). The effect of retention interval on the eyewitness identification confidence-accuracy relationship. Journal of Applied Research in Memory and Cognition, 5(2), 192-203. doi: 10.1016/j.jarmac.2016.04.006

Woolnough, P. S., \& MacLeod, M. D. (2001). Watching the birdie watching you: Eyewitness memory for actions using CCTV recordings of actual crimes. Applied Cognitive Psychology, 15, 395-411. doi: 10.1002/acp.717

Yerkes, R. M., \& Dodson, J. D. (1908). The relation of strength of stimulus to rapidity of habitinformation. Journal of Comparative Neurology of Psychology, 18, 459-482. doi:

$10.1002 /$ cne. 920180503

Yuille, J. C. (2013). The challenge for forensic memory research: Methodolotry. In B. S. Cooper, D. Griesel, \& M. Ternes, Applied Issues in Investigative Interviewing, Eyewitness Memory, and Credibility Assessment (pp. 3-18). Springer, New York, NY. doi: 10.1007/978-1-46145547-9_1 
Tabela 1

Níveis de Acurácia e Confiança para Memória do Crime Entre as Condições de Carga Emocional

\begin{tabular}{lcccccc}
\hline & Controle & $\begin{array}{c}\text { Menor Carga } \\
\text { Emocional } \\
M(D P)\end{array}$ & $\begin{array}{c}\text { Maior Carga } \\
\text { Emocional } \\
M(D P)\end{array}$ & $F$ & $p$ & $\eta^{2}$ p \\
\hline $\begin{array}{l}\text { Acurácia } \\
\quad \text { Informações Gerais }\end{array}$ & $26.97(2.21)$ & $27.1(2.41)$ & $27.47(1.72)$ & 0.443 & .64 & 0.010 \\
$\quad \begin{array}{l}\text { Informações } \\
\quad \text { Centrais }\end{array}$ & $13.23(1.43)$ & $13.33(1.61)$ & $13.47(1.23)$ & 0.204 & .81 & 0.003 \\
$\quad \begin{array}{l}\text { Informações } \\
\text { Periféricas }\end{array}$ & $13.73(1.01)$ & $13.77(1.07)$ & $14.00(0.95)$ & 0.617 & .54 & 0.015 \\
$\begin{array}{l}\text { Confiança } \\
\quad \text { Informações Gerais }\end{array}$ & $81.29(13.01)$ & $81.02(11.33)$ & $82.73(10.03)$ & 0.191 & .82 & 0.004 \\
$\quad \begin{array}{l}\text { Informações } \\
\quad \text { Centrais }\end{array}$ & $80.54(13.09)$ & $80.10(11.17)$ & $82.63(11.46)$ & 0.384 & .68 & 0.008 \\
$\quad \begin{array}{l}\text { Informações } \\
\quad \text { Periféricas }\end{array}$ & $82.09(15.24)$ & $82.00(12.95)$ & $82.86(12.98)$ & 0.035 & .96 & 0.001 \\
\hline
\end{tabular}


Tabela 2

Níveis de Acurácia e Confiança para Tarefa de Identificação por Fotos Entre as Condições de Carga Emocional

\begin{tabular}{lcccccc} 
& Controle & $\begin{array}{c}\text { Menor Carga } \\
\text { Emocional } \\
M(D P)\end{array}$ & $\begin{array}{c}\text { Maior Carga } \\
\text { Emocional } \\
M(D P)\end{array}$ & $F$ & $p$ & $\eta^{2} \mathrm{p}$ \\
\hline $\begin{array}{l}\text { Primeiro conjunto de } \\
\text { fotos }\end{array}$ & $0.53(0.51)$ & $0.53(0.51)$ & $0.43(0.50)$ & 0.390 & .67 & 0.008 \\
$\begin{array}{l}\text { Segundo conjunto de } \\
\text { fotos }\end{array}$ & & & & & \\
$\quad$ Acurácia Geral & $0.52(0.51)$ & $0.40(0.50)$ & $0.30(0.47)$ & 1.445 & .24 & 0.032 \\
$\quad$ Alvo presente & $0.14(0.36)$ & $0.20(0.41)$ & $0.07(0.26)$ & 0.545 & .58 & 0.025 \\
$\quad$ Alvo ausente & $0.87(0.35)$ & $0.60(0.51)$ & $0.53(0.52)$ & 2.162 & .12 & 0.09 \\
\hline
\end{tabular}


Tabela 3

Níveis de Acurácia e Confiança para Memória do Crime Entre as Condições de Intervalo de Retenção

\begin{tabular}{|c|c|c|c|c|c|c|}
\hline & 7 Dias & 14 Dias & 21 Dias & $F$ & $p$ & $\eta_{p}^{2}$ \\
\hline & $M(D P)$ & $M(D P)$ & $M(D P)$ & & & \\
\hline \multicolumn{7}{|l|}{ Acurácia } \\
\hline Informações Gerais & $24.00(2.42)$ & $22.95(2.94)$ & $22.45(2.18)$ & 1.592 & .21 & 0.055 \\
\hline $\begin{array}{l}\text { Informações } \\
\text { Centrais }\end{array}$ & $12.14(1.56)$ & $11.48(2.04)$ & $11.32(1.49)$ & 1.030 & .36 & 0.035 \\
\hline $\begin{array}{l}\text { Informações } \\
\text { Periféricas }\end{array}$ & $11.86(1.70)$ & $11.48(1.89)$ & $11.14(1.98)$ & 0.633 & .53 & 0.022 \\
\hline \multicolumn{7}{|l|}{ Confiança } \\
\hline Informações Gerais & $76.24(12.92)$ & $65.28(16.44)$ & $61.00(17.84)$ & 3.834 & .02 & 0.124 \\
\hline $\begin{array}{l}\text { Informações } \\
\text { Centrais }\end{array}$ & $74.15(13.41)$ & $61.90(19.82)$ & $55.60(21.11)$ & 4.084 & .02 & 0.131 \\
\hline $\begin{array}{l}\text { Informações } \\
\text { Periféricas }\end{array}$ & $78.48(14.81)$ & $68.89(15.36)$ & $66.76(17.21)$ & 2.454 & .09 & 0.083 \\
\hline
\end{tabular}


Tabela 4

Níveis de Acurácia e Confiança para Tarefa de Identificação por Fotos Entre as Condições de Intervalo de Retenção

\begin{tabular}{lcccccc}
\hline & $\begin{array}{c}\text { Dias } \\
M(D P)\end{array}$ & $\begin{array}{c}14 \text { Dias } \\
M(D P)\end{array}$ & $\begin{array}{c}21 \text { Dias } \\
M(D P)\end{array}$ & $F$ & $p$ & $\eta^{2}$ p \\
\hline $\begin{array}{l}\text { Primeiro conjunto de } \\
\text { fotos }\end{array}$ & $0.43(0.51)$ & $0.24(0.44)$ & $0.36(0.49)$ & 0.739 & .48 & 0.026 \\
$\begin{array}{l}\text { Segundo conjunto de } \\
\text { fotos }\end{array}$ & & & & & \\
$\quad$ Acurácia Geral & $0.50(0.52)$ & $0.33(0.48)$ & $0.32(0.48)$ & 0.676 & .51 & 0.024 \\
$\quad$ Alvo presente & $0.00(0.00)$ & $0.00(0.00)$ & $0.08(0.28)$ & 0.638 & .53 & 0.045 \\
$\quad$ Alvo ausente & $0.88(0.35)$ & $0.70(0.48)$ & $0.67(0.50)$ & 0.507 & .61 & 0.04 \\
\hline
\end{tabular}




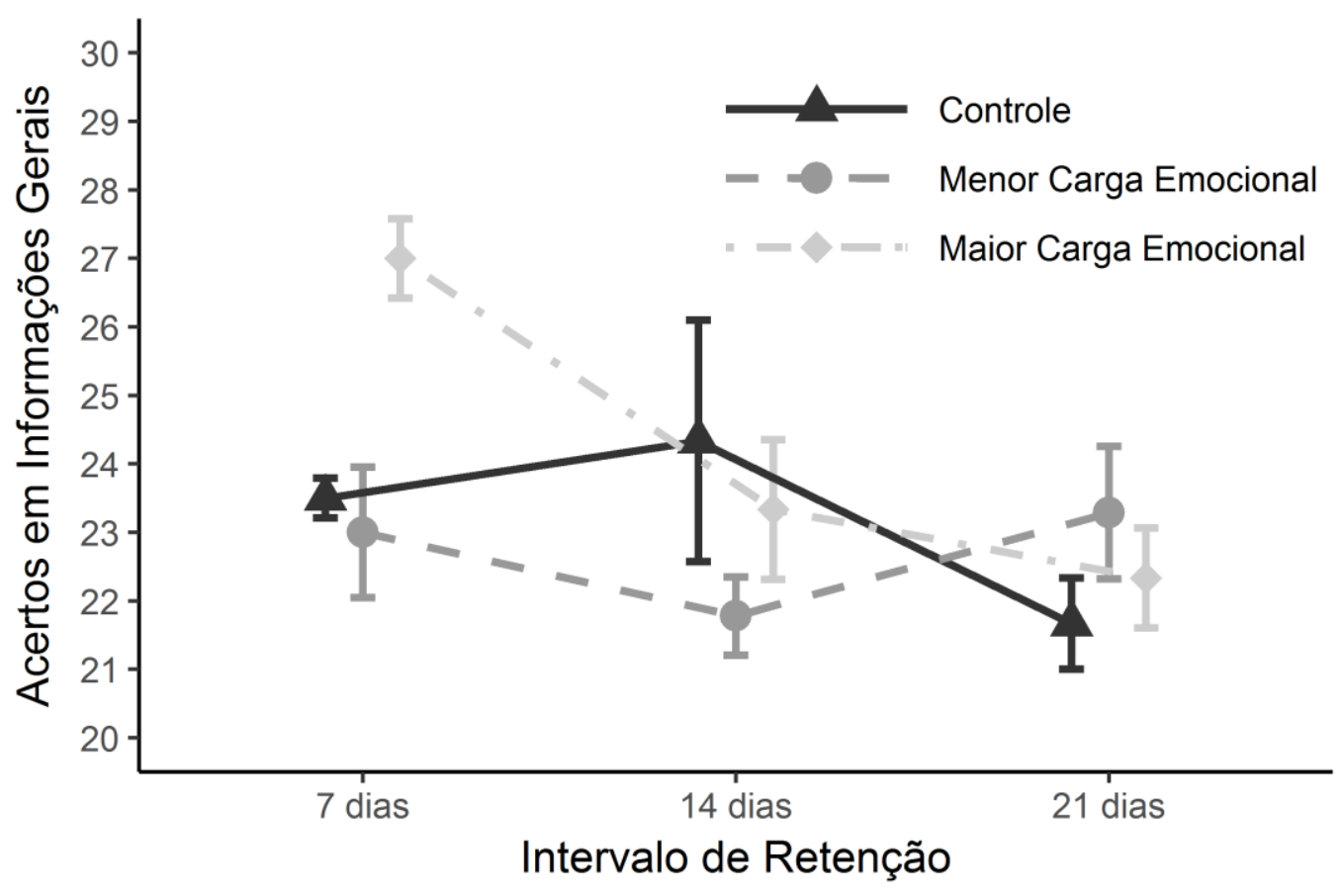

Figura 1. Acurácia para informações gerais sobre o crime, por condições de carga emocional e intervalo de retenção 


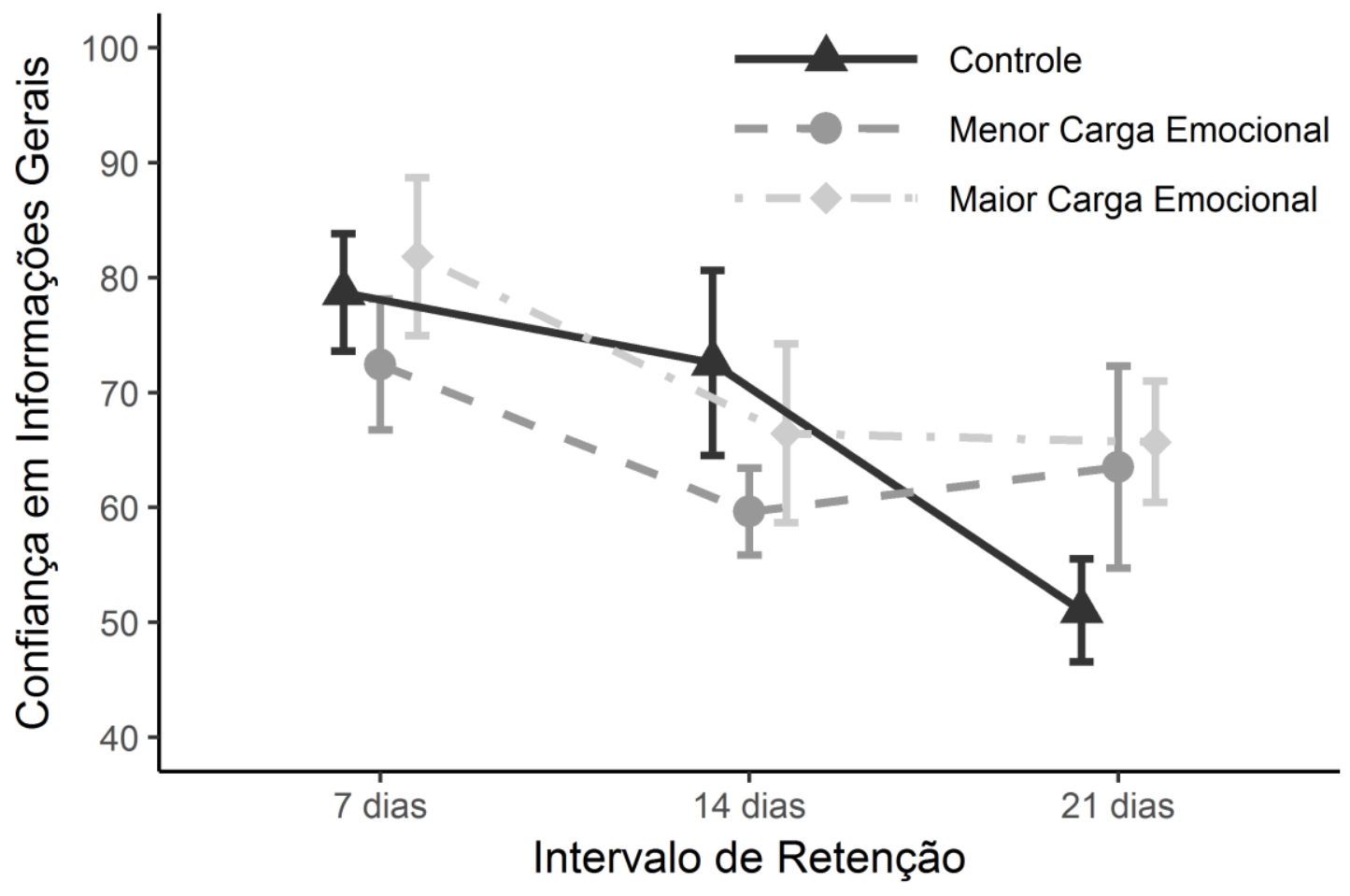

Figura 2. Confiança para informações gerais sobre o crime, por condições de carga emocional e intervalo de retenção 